\title{
REFLECTION AND DISSIPATION OF OBLIQUE ALFVÉN WAVES IN AN ISOTHERMAL ATMOSPHERE
}

\author{
HADI YAHYA ALKAHBY and M. A. MAHROUS
}

(Received 25 April 1996)

\begin{abstract}
In this article, we investigate the combined effects of viscosity and Ohmic electrical conductivity on upward and downward propagation oblique Alfvén waves in an isothermal atmosphere. It is shown that the presence and direction of the magnetic field play an important role in the structure and the heating mechanism of solar atmosphere. In addition, the atmosphere can be divided into two distinct regions connected by a transition region. In the lower region, the solution can be written as a linear combination of an upward and a downward propagation wave with unequal wavelengths. In the upper region, the solution decays exponentially with the altitude. Moreover, the magnetic field creates a reflecting and a non-absorbing transition region. On the contrary, the viscosity and Ohmic electrical conductivity produce a reflecting and an absorbing transition region. The nature of the transition region depends on the relative strength of the viscous diffusivity with respect to the resistive diffusivity and on the direction of the magnetic field. A unique solution is determined. The reflection coefficient and damping factors are derived and the conclusions are discussed in connection with the nature of the heating mechanism of the solar atmosphere.
\end{abstract}

Keywords and phrases. Atmospheric waves, Alfvén waves, wave propagation.

1991 Mathematics Subject Classification. 76N, 76Q.

1. Introduction. The propagation of atmospheric waves in an isothermal atmosphere has been studied in recent years. The motivation for these studies comes from their applications to phenomena in compressible ionized fluids such as solar, stellar, and earth's atmosphere. The presence of dissipation in an isothermal atmosphere may cause upward travelling small amplitude waves to be reflected downward. This type of reflection is most significant when the wavelength is large compared to the density scale height. In addition, the dynamic of the solar atmosphere is complicated by the fact that not only is it strongly stratified in both gas density and temperature but it is, also, permeated by a non-uniform magnetic field. The solar atmosphere is an example of a plasma that is both structured and stratified. Structuring because of the powerful ordering of the magnetic field-lines that emerge through the photosphere into concentrated flux tubes. This indicates that the direction of the magnetic field plays an important role in the heating process of the solar atmosphere. In particular, the heating mechanism of the solar atmosphere depends mainly on the direction and strength of the magnetic field. Thus, the presence of dissipative factors, such as viscosity and Ohmic electrical conductivity, in an oblique magnetic field may explain certain aspects of the heating process of the solar atmosphere.

The aim of the present work is to examine the reflection and dissipation of oblique 
Alfvén waves in a viscous and resistive isothermal atmosphere. It is shown that when the viscosity, resistivity or the magnetic field dominates the oscillatory process, the atmosphere can be divided into two distinct regions. These two regions are connected by a transition region in which the reflection, dissipation of the energy and modification of the solar waves take place. The nature of the transition region indicates that the tunneling of the wave is very weak because of the presence of viscosity and Ohmic electrical conductivity. There is no tunneling when the magnetic field is horizontal and dominate the oscillatory process. The reflection, dissipation and the nature of the transition region depend on the relative strength of the viscous diffusivity with respect to the resistive diffusivity and on the direction of the magnetic field. As a result, we have the following cases:

(a) when the viscosity dominates the oscillatory process, the solution that satisfies the prescribed boundary conditions, can be written as a linear combination of an incident and a reflected wave, in the lower region, with equal wavelength. In the upper region the solution decays exponentially with altitude. At the same time, the transition region acts like a reflecting and absorbing layer;

(b) when the electrical diffusivity dominates the motion, the behavior of the solution in the upper region and in the transition region stays the same whereas, in the lower region, the energy of the incident and reflected waves dissipates by unequal rate of dissipation;

(c) when the magnetic field dominates the motion, the transition region acts approximately as a non-absorbing but a reflecting layer. In addition, the magnitude of the reflection coefficients tends to one as the angle between the direction of the magnetic field and the vertical tends to zero. Thus, the presence and direction of the magnetic field play an important role in the structure and heating process of the solar atmosphere.

The problem can be reduced to the hypergeometric differential equations with three regular singular points. The middle regular singular point represents the existence and approximate location of the transition region. The reflection coefficient, dissipative factors and wave numbers are determined and the model is investigated in connection with the heating process of the solar atmosphere.

2. Statement of the problem. We consider the effect of the viscosity and Ohmic electrical conductivity on the reflection and dissipation of oblique Alfvén waves, i.e., magnetic waves that resulted from an oblique magnetic field. The atmosphere under consideration is assumed to be isothermal with density $\rho(z)$ which depends only on the altitude $z$ and under the influence of a uniform horizontal magnetic field $\mathbf{B}(z)=$ $B(z) \mathrm{e}_{x}$ and an oblique magnetic field $\mathbf{B}=B(z)\left[\sin \varphi \mathrm{e}_{x}+\cos \varphi \mathrm{e}_{z}\right]$, where $\varphi$ is the angle of the magnetic field with the vertical axis $z$. The perturbation quantities of the velocity and magnetic field strength are denoted by $\mathrm{v}(\mathrm{x}, t)=v(x, z, t) \mathrm{e}_{y}$ and $\mathrm{h}(\mathrm{x}, t)=$ $h(x, z, t) \mathrm{e}_{y}$, respectively. The total magnetic field is:

$$
\mathbf{H}(\mathrm{x}, t)=B\left[\sin \varphi \mathrm{e}_{x}+h(x, z, t) \mathrm{e}_{y}+\cos \varphi \mathrm{e}_{z}\right] .
$$

We assume that the velocity of the wave and the magnetic field perturbation are orthogonal to the $(x, z)$ plane of gravity $g$. The equations of momentum and induction 
are:

$$
\begin{aligned}
\frac{\partial v}{\partial t}-\alpha^{2} \nabla_{\phi} h & =\eta \nabla^{2} v \\
\frac{\partial h}{\partial t}-\nabla_{\phi} v & =\kappa \nabla^{2} h-\frac{\partial \kappa}{\partial z} \cdot \frac{\partial h}{\partial z},
\end{aligned}
$$

where

$$
\nabla^{2}=\frac{\partial^{2}}{\partial x^{2}}+\frac{\partial^{2}}{\partial z^{2}} \quad \text { and } \quad \nabla_{\phi}=\sin \varphi \frac{\partial}{\partial x}+\cos \varphi \frac{\partial}{\partial z} .
$$

The parameter $\eta$ denotes the viscosity coefficient, while the parameters $\alpha^{2}$, and $\kappa$ denote Alfvén speed, and resistive diffusity, both are dependent on $z$ and can be written as:

$$
\begin{aligned}
\alpha^{2}(z) & =\frac{\mu B^{2}}{4 \pi \rho(z)}, \\
\kappa(z) & =\frac{c^{2}}{4 \pi \sigma(z)},
\end{aligned}
$$

where $\mu$ is the magnetic permeability, $c$ is the speed of light, and $\sigma$ is the Ohmic electrical conductivity. We consider the case of uniform $\kappa$ and $\eta$, i.e., $\frac{d \kappa}{d z}=\frac{d \eta}{d z}=0$. The differential for the velocity $v$ can be obtained by differentiating equation (2.2) with respect to $t$ and using equation (2.3):

$$
\frac{\partial^{2} v}{\partial t^{2}}-\alpha^{2} \nabla_{\phi}^{2} v=\left[\left(\eta \nabla^{2}+\alpha^{2} \kappa \nabla^{2} \alpha^{-2}\right) \frac{\partial}{\partial t}-\alpha^{2} \kappa \nabla^{2}\left(\alpha^{-2} \eta\right) \nabla^{2}\right] v
$$

Since the atmosphere is steady and horizontally homogeneous, we can use Fourier decomposition in time $t$ and the horizontal coordinate $x$ :

$$
v(x, z, t)=\iint_{-\infty}^{\infty} V(z, k, \omega) \exp [i(k x-\omega t)] d k d \omega,
$$

where $V(z, k, \omega)$ denotes the velocity perturbation spectrum of waves of frequency $\omega$ and horizontal wave number $k$ at altitude $z$. As a result, the differential equation (2.7) can be written in the following form:

$$
\begin{aligned}
& {\left[\left(\alpha^{2} \cos ^{2} \varphi-i \omega(\kappa+\eta)\right) D^{2}+2 i\left(k \alpha^{2} \sin \varphi \cos \varphi+2 \omega \kappa(D \alpha / \alpha)\right) D\right] V} \\
& \quad+\left[\omega^{2}-k^{2} \alpha^{2} \sin ^{2} \varphi+i \omega k^{2}(\kappa+\eta)+\eta \omega \chi\left(D^{2} \alpha / \alpha-3(D \alpha)^{2} / \alpha^{2}\right)\right] V=0,
\end{aligned}
$$

where $D$ denote differentiation with respect to $z$.

BOUNDARY CONDITIONS. The oscillations can be assumed to be initiated at $z=0$ or lower. The exact nature of the mechanism of excitation is not considered since our aim is to investigate the reflection and dissipation phenomenon at high altitude. At $z=0$, we assume that:

$$
V(0, k, t)=1,
$$

by suitable normalization of $V(z, k, t)$. Physically relevant solutions must also satisfy one of the following conditions: 


$$
\eta \int|D V|^{2} d z<\infty, \quad \kappa \int|D V|^{2} d z<\infty .
$$

The boundary conditions in (2.11) are called upper boundary conditions. They are applicable if $\eta \neq 0$ or $\kappa \neq 0$ and are not applicable if $\eta=\kappa=0$. It is seen that the boundary conditions (2.10) and (2.11) determine a unique solution for the boundary value problem defined by the differential equation (2.9).

3. Series and asymptotic solutions. To obtain series and asymptotic solutions of the boundary value problem (2.9) satisfying the boundary conditions (2.10) and (2.11), certain assumptions and simplifications are needed. The equilibrium pressure $P_{0}$, density $\rho_{0}$ and temperature $T_{0}$ satisfy the gas law $P_{0}=R T_{0} \rho_{0}$ and the hydrostatic equation $P_{0}^{\prime}+g \rho_{0}$. Here $R$ is the gas constant, $g$ is the gravity acceleration and the prime " ' ' denotes differentiation with respect to $z$. The equilibrium pressure and density can be written as:

$$
P_{0}(z)=P_{0}(0) \exp \left(-\frac{z}{H}\right), \quad \rho_{0}(z)=\rho_{0}(0) \exp \left(-\frac{z}{H}\right),
$$

where $H=R T_{0} / g$ is the density scale height. As a result, we have:

$$
\alpha^{2}=\alpha_{0}^{2} \exp \left(\frac{z}{H}\right), \quad \eta(z)=\eta_{0} \exp \left(\frac{z}{H}\right),
$$

where $\alpha_{0}^{2}=\mu B^{2} / 4 \pi \rho_{0}(0)$. Since considering an isothermal atmosphere, the electrical diffusivity $\kappa(z)$, which depends mainly on the temperature and rate of ionization, is constant, i.e., $\kappa(z)=\kappa_{0}$. Moreover, it is convenient to write equation (2.9) in dimensionless form. To do this, we introduce the following dimensionless quantities:

$$
z^{\prime}=\frac{z}{H}, \quad \omega^{\prime}=\frac{\omega H}{\alpha_{0}}, \quad k^{\prime}=k H, \quad \beta_{1}=\frac{\eta_{0} \omega}{\alpha_{0}^{2}}, \quad \beta_{2}=\frac{\chi_{0} \omega}{\alpha_{0}^{2}} .
$$

The primes are omitted since all quantities are dimensionless from now on. The differential equation (2.9) can be written as:

$$
\begin{aligned}
& {\left[\cos ^{2} \varphi-i\left[\beta_{1}+\beta_{2} \exp (-z)\right]\right] D^{2} V+2 i\left[k \cos \varphi \sin \varphi+\beta_{2} \exp (-z)\right] D V} \\
& +\left[k^{2}\left(i \beta_{2}-\sin ^{2} \varphi\right)+\left[\omega^{2}+i \beta_{2}\left(k^{2}-1\right)\right] \exp (-z) V\right]=0 .
\end{aligned}
$$

Introduce a new dimensionless variable $\xi$ defined by

$$
\xi(z)=\frac{\exp (-z)}{\varepsilon}, \text { where } \varepsilon=\frac{\cos ^{2} \varphi-i \beta_{1}}{i \beta_{2}},
$$

and let

$$
V(z, k, \omega)=\xi^{q} \chi(\xi),
$$

where $q$ is a parameter that determines the asymptotic behavior of the solution at high altitude. The value of the parameter $q$ is chosen so that the coefficient of $\xi$ does not depend on $q$. As a result, the parameter $q$ should satisfy the relation

$$
\beta_{3} q^{2}-\beta_{4} q-\beta_{5}=0
$$


where

$$
\beta_{3}=\cos ^{2} \varphi-i \beta_{1}, \quad \beta_{4}=2 i k \cos \varphi \sin \varphi, \quad \beta_{5}=k^{2}\left(\sin ^{2} \varphi-i \beta_{1}\right) .
$$

Consequently, the differential equation (3.4) can be written, using, (3.5), (3.6), (3.7), and (3.8), in the following form:

$$
\xi(1-\xi) \frac{d^{2} \chi}{d \xi}+\left[1+2 q-\beta_{4} / \beta_{3}-(3+2 q) \xi\right] \frac{d \chi}{d \xi}-\left[(q+1)^{2}-k^{2}+i \omega^{2} / \beta_{2}\right] \chi=0 .
$$

The differential equation (3.9) is a special case of the hypergeometric equation

$$
\xi(1-\xi) \frac{d^{2} \chi}{d \xi}+[c-(a+b+1) \xi] \frac{d \chi}{d \xi}-a b x=0,
$$

where the parameters $a, b$, and $c$ satisfy the following relations:

$$
c=1+q-\frac{\beta_{4}}{\beta_{3}}, \quad a+b=2(1+q), \quad a b=(q+1)^{2}-k^{2}+i \frac{\omega^{2}}{\beta_{2}} .
$$

Solving for the parameters $a$ and $b$, using equation (3.7), one obtains:

$$
a=D_{a}+i W_{a}, \quad b=D_{b}-i W_{b} .
$$

The damping factors $D_{a}$ and $D_{b}$ are defined by:

$$
\begin{aligned}
& D_{a}=1-\frac{1}{s}\left[k\left[r_{2} \cos \varphi+\beta_{1}\left(r_{1}+\cos \varphi \sin \varphi\right)\right]+k r_{3} \cos ^{2} \varphi+r_{4} \beta_{1}\right], \\
& D_{b}=1+\frac{1}{s}\left[r_{3} \cos ^{2} \varphi+r_{4} \beta_{1}-k\left[r_{2} \cos ^{2} \varphi+\beta_{1}\left(r_{1}+\cos \varphi \sin \varphi\right)\right]\right] .
\end{aligned}
$$

The parameters $W_{a}$ and $W_{b}$ denote wave numbers and are defined by:

$$
\begin{aligned}
W_{a} & =\frac{1}{s}\left[k\left(\cos ^{3} \varphi \sin \varphi+r_{1} \cos ^{2} \varphi+r_{2} \beta_{1}\right)+r_{4} \cos ^{2} \varphi-r_{3} \beta_{1}\right], \\
W_{b} & =\frac{1}{s}\left[r_{4} \cos ^{2} \varphi+k r_{2} \beta_{1}-r_{3} \beta_{1}-k\left(\cos ^{3} \varphi \sin \varphi+r_{1} \cos ^{2} \varphi\right)\right], \\
s & =\cos ^{4} \varphi+\beta_{1},
\end{aligned}
$$

where $r_{1}$ and $r_{2}$ are the real and imaginary parts of $\sqrt{\beta_{1}^{2}+i \beta_{2}}, r_{3}$ and $r_{4}$ are the real and imaginary parts of $\sqrt{k^{2}-i \omega^{2} / \beta_{2}}$.

It is clear that the differential equation (3.9) has three regular singular points at $\xi=0, \xi=1$, and $\xi=\infty$. Also, since none of the parameters $c, c-a-b$, and $a-b$ is an integer, equation (3.9) has two linearly independent solutions in the neighborhood of $\xi=0$, i.e., $|\xi|<1$, which can be written in the following form:

$$
\begin{aligned}
& \chi_{1}(\xi)=F(a, b ; c ; \xi), \\
& \chi_{2}(\xi)=\xi^{1-c} F(a-c+1, b-c+1 ; 2-c ; \xi),
\end{aligned}
$$

where $F(a, b ; c ; \xi)$ is the hypergeometric function defined by

$$
F(a, b ; c ; \xi)=\frac{\Gamma(c)}{\Gamma(a) \Gamma(b)} \sum_{0}^{\infty} \frac{\Gamma(a+n) \Gamma(b+n)}{\Gamma(c+n)} \frac{\xi^{n}}{n !} .
$$


By simple computations, we find that

$$
\begin{aligned}
1-c & =-c_{1}+i c_{2}, \\
c_{1} & =\frac{k}{s}\left[r_{2} \cos ^{2} \varphi+\beta_{1}\left(r_{1}-\cos \varphi \sin \varphi\right)\right], \\
c_{2} & =\frac{k}{s}\left[-r_{2} \cos ^{2} \varphi+\beta_{1} r_{2}+\cos ^{2} \varphi \sin \varphi\right] .
\end{aligned}
$$

It is clear that $c_{1}>0$. Consequently, using equation (3.5), the solution $\chi_{2}(\xi)$ increases exponentially with the altitude $z$. As a result, the solution $\chi_{2}(\xi)$ does not satisfy the upper boundary condition because of the dissipation conduction (2.11) while the solution $\chi_{1}(\xi)$ decreases exponentially with altitude. From this, we conclude that the solution of the differential equation (3.9) can be written in the following form:

$$
\chi(\xi)=C \chi_{1}(\xi)=C F(a, b ; c ; \xi) .
$$

The constant $C$ is determined by the lower boundary condition (2.10).

4. Magnitude of the reflection coefficient. From equation (3.5), it is clear that $|\arg (-\xi)|<\pi$. As a result, the solution of the differential equation (3.9), given in (3.24), for $|\xi|>1$ as $\epsilon \rightarrow 0$, can be written as:

$$
x(\xi)=C\left[\begin{array}{c}
\frac{\Gamma(c) \Gamma(b-a)}{\Gamma(b) \Gamma(c-a)}(-\xi)^{-a} F\left(a, 1-c+a ; 1-b+a ; \frac{1}{s}\right) \\
+\frac{\Gamma(c) \Gamma(a-b)}{\Gamma(a) \Gamma(c-b)}(-\xi)^{-b} F\left(b, 1-c+b ; 1-a+b ; \frac{1}{s}\right)
\end{array}\right] .
$$

Retaining the most significant terms in equation (4.1), we obtain:

$$
\chi(\xi)=C\left[\frac{\Gamma(c) \Gamma(b-a)}{\Gamma(b) \Gamma(c-a)}(-\xi)^{-a}+\frac{\Gamma(c) \Gamma(a-b)}{\Gamma(a) \Gamma(c-b)}(-\xi)^{-b}\right] .
$$

Reintroducing the dimensionless variable $z$ via equation (3.5), equation (4.2) becomes

$$
\chi(z)=C A\left[\exp \left(D_{a}+i W_{a}\right) z+R_{c} \exp \left(D_{b}-i W_{b}\right) z\right]
$$

where

$$
A=\frac{\Gamma(c) \Gamma(b-a)}{\Gamma(b) \Gamma(c-a)} \exp \left[\left(D_{a}+i W_{a}\right)\left(\log \varepsilon+i \theta_{\epsilon}\right)\right],
$$

and $\theta_{\epsilon}=\arg (\varepsilon)$. The reflection coefficient $R_{\mathcal{C}}$ is defined by:

$$
R_{c}=\frac{\Gamma(b) \Gamma(a-b) \Gamma(c-a)}{\Gamma(a) \Gamma(b-a) \Gamma(c-b)} \exp \left[(a-b)\left(\log \varepsilon+i \theta_{\epsilon}\right)\right] .
$$

The constant $C$ is determined from equation (4.4) using the boundary condition (2.10). As a result, we have:

$$
C=\frac{1}{A\left(1+R_{c}\right)}
$$

Consequently, the solution of the differential equation (3.9), below the reflecting layer, which satisfies the prescribed boundary conditions can be written in the following form:

$$
\chi(z) \sim \frac{1}{\left(1+R_{c}\right)}\left[\exp \left(D_{a}+i W_{a}\right) z+R_{c} \exp \left(D_{b}-i W_{b}\right) z\right]
$$


5. Conclusions and general remarks. It is clear that the parameters $D_{a}, D_{b}, W_{a}$, and $W_{b}$ depend on the viscosity, resistivity, and $\varphi$. Thus, the study of the reflection and dissipation of the solar waves and any comparison among the effects of these parameters depends on the relative strength of the viscous and resistive diffusivities and the direction of the magnetic field. As a result, we have the following conclusions:

[I] Equation (4.7) represents the behavior of the solution of the differential equation (3.9), that satisfies the prescribed boundary conditions, below the reflecting layer and indicates that the solution can be written as a linear combination of an upward and a downward propagating wave. It is clear that $D_{a} \neq D_{b}$ and $W_{a} \neq W_{b}$. This indicates that the wavelength of incident wave does not equal the wavelength of the reflected wave, which shows that the resonance cannot take place. In addition, the rate at which the energy of the incident wave dissipates is different from that of the reflected one. This shows that the dissipation of the energy of the wave takes place not only in the transition layer but also below the reflecting layer as the waves propagate upward and downward. Above the reflecting layer, the solution decays exponentially with altitude. The dissipated energy, above, below, and in the reflecting layer, contributes to the heating of the solar atmosphere.

[II] The regions, below and above the reflecting layer, are connected by a transition region which is located in the vicinity of $|\varepsilon| e^{z} \longrightarrow 1$. In the transition region, the wave modification and most of its energy dissipation take place. The nature of dissipation in the transition region depends on the ratios of viscous and resistive diffusivities and on the direction of the magnetic field. The presence of the transition region is essential not only for the dissipation of the energy of the wave but also for the reflection process. The transition region behaves as follows:

(a) when the effect of the viscosity and resistivity is negligible compared to that of the magnetic field, the transition region acts like a reflecting and non-absorbing layer;

(b) when either the effect of the viscosity or that of the resistivity is large compared to that of the magnetic field, the transition layer acts as an absorbing and a reflecting layer.

[III] One of the simplest models of electrical conduction in ionized gases considers only the current due to electrons, i.e., we neglect the motion of positive ions and neutrals because they are heavier and slower in their motion. As a result, the electrical current and inertia forces are:

$$
\mathbf{J}=N e \mathbf{V}, \quad e \mathbf{E}=m \frac{d \mathbf{V}}{d \tau},
$$

where $N$ is the unit volume, $e$ is the electron charge, $\mathbf{E}$ is the electric field, and $\tau$ is the mean time between colission charges inversely with the electron density if the atmosphere is fully ionized. If the rate of ionization is constant, the Ohmic electrical conductivity is approximately independent of the mass density. As a result, the Ohmic electrical conductivity is constant in an isothermal atmosphere. Consequently, the dissipation in only viscous atmosphere takes place mainly in the transition region. In only a resistive atmosphere, the energy of the wave dissipates as it propagates below the reflecting layer and in the transition region. This leads to intense heating process in the solar atmosphere. 
[IV] The argument in [III] justifies the necessity of the dissipation condition. Moreover, the combined effect of the viscosity and resistivity explains the way in which the heating process of the solar atmosphere takes place and the difference in the amount of heat in different regions with the same magnetic field. In addition, since the reflection coefficient and the approximate location of the transition region depend on the ratio of the viscosity with respect to the resistivity and the angle of the inclination of the magnetic field, the direction of the magnetic field influences the propagation of the waves in all regions and even if $\kappa=\eta=0$, the magnitude of the reflection coefficient is not always equal to one. This is due to the direction in which the wave travels. For an oblique magnetic field, as it tilts closer to the horizontal, the altitude of the transition region increases until it recedes to infinity for a horizontal field. In this case, the lower region fills the whole atmosphere. This can be explained by the fact that the lack of a vertical component of the external magnetic field does not favor either upward or downward propagation and favors the dissipation along the horizontal direction.

[V] It is clear that the differential equation (3.9) has three regular singular points. They are $\xi=\xi_{1}=0, \xi=\xi_{2}=1$, and $\xi=\xi_{3}=\infty$. The middle regular singular point represents the approximate location of the transition region. As $\xi_{2} \longrightarrow \xi_{3}$, the transition region recedes to infinity, but the case of $\eta \longrightarrow \kappa \rightarrow 0$ will not be recovered because no matter how small the diffusivities and the Alfvén speed are, they create a reflecting and dissipating layer and we have a solution that can be written as a linear combination of an upward and downward propagating wave. As the transition region recedes to infinity, the two regular singular points $\xi_{2}$, and $\xi_{3}$ coincide which leads to an irregular singular point, whereas the singularity $\xi_{1}=0$ of the equation remains regular. As a result, the solution of the differential equation (3.9) is given in the following cases:

(a) In the case of a non-horizontal magnetic field, which is the subject of this paper, the solution can be expressed in terms of the hypergeometric functions, with three regular singular points. The intermediate singular point specifies the existence and location of the transition region where the motion of the waves is dominated by viscous and resistive dissipation;

(b) In the case of the horizontal magnetic field, the solution can be expressed in terms of a confluent hypergeometric function with a regular singular point at $\xi=1$ and irregular one at $\xi=\xi_{2}=\xi_{3}=\infty$. As a result, we have one region, in which the solution can be written as a linear combination of an upward and a downward propagating wave which is dominated by dissipation. Also, in this case, the solution may be expressed in terms of Bessel Functions.

ACKNOWLEDGEMENT. The authors would like to express their sincere thanks to Professor Michael Yanowitch for his support and invaluable criticism during the preparation of this work.

\section{REFERENCES}

[1] M. Abramowitz and I. A. Stegun, Handbook of mathematical functions with formulas, graphs, and mathematical tables, National Bureau of Standards Applied Mathematics Series, vol. 55, For sale by the Superintendent of Documents, U.S. Government Printing Office, Washington, 1964. MR 29\#4914. Zbl 171.38503. 
[2] H. Alkahby and M. Yanowitch, Reflection of vertically propagating waves in a thermally conducting isothermal atmosphere with a horizontal magnetic field, Geophys. Astrophys. Fluid Dynam. 56 (1991), 227-235.

[3] H. Y. Alkahby, On the coronal heating mechanism by the resonant absorption of Alfven waves, Internat. J. Math. Math. Sci. 16 (1993), no. 4, 811-816. CMP 9317. Zbl 779.76102.

[4] H. Y. Alkahby, M. Mahrous, and L. Debnath, The effect of Newtonian cooling on the reflection and dissipation of hydromagnetic waves in a viscous and thermally conducting isothermal atmosphere, Geophys. Astrophys. Fluid Dynam. 81 (1995), 57-71.

[5] M. Yanowitch, Effect of viscosity on gravity waves and the upper boundary conditions, J. Fluid Mech. 29 (1967), 209-231. Zbl 149.45703.

AlKahby: Department of MATHEMATics AND Computer SCIENCE, Dillard University, NEW ORLEANS, LA 70122, USA

MAHROUS: DEPARTMENT OF MATHEMATICS, UNIVERSITY OF NEW ORLEANS, NEW ORLEANS, LA 70148, USA 


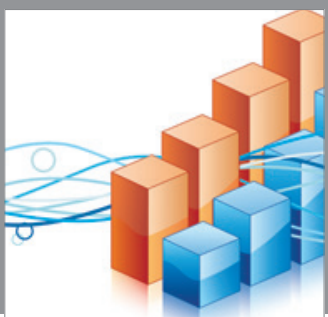

Advances in

Operations Research

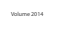

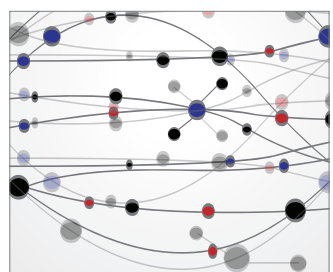

\section{The Scientific} World Journal
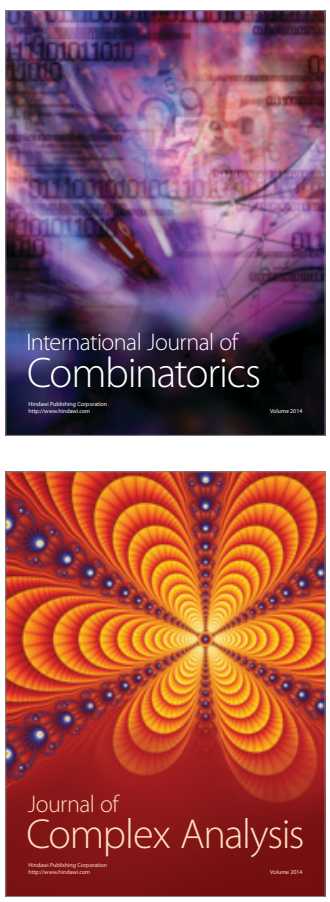

International Journal of

Mathematics and

Mathematical

Sciences
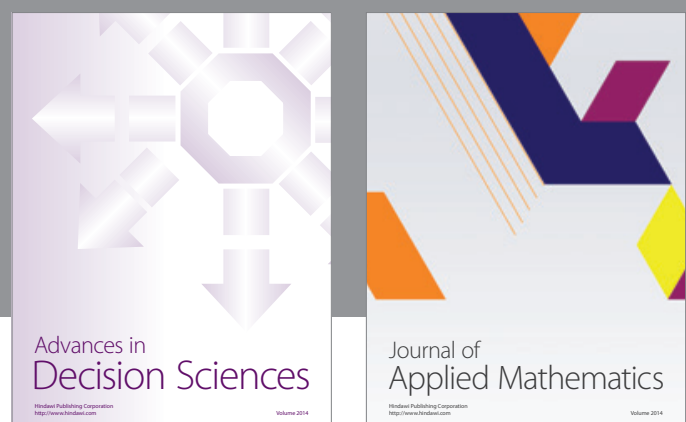

Journal of

Applied Mathematics
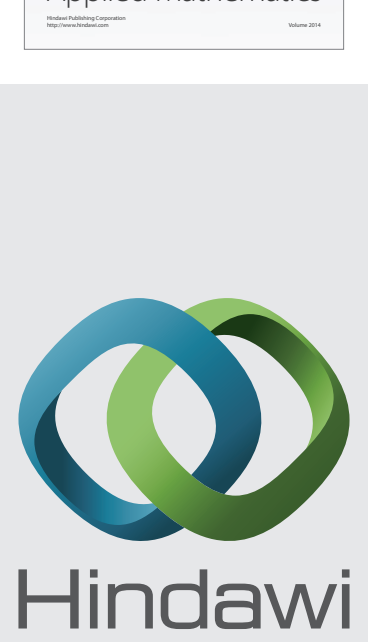

Submit your manuscripts at http://www.hindawi.com
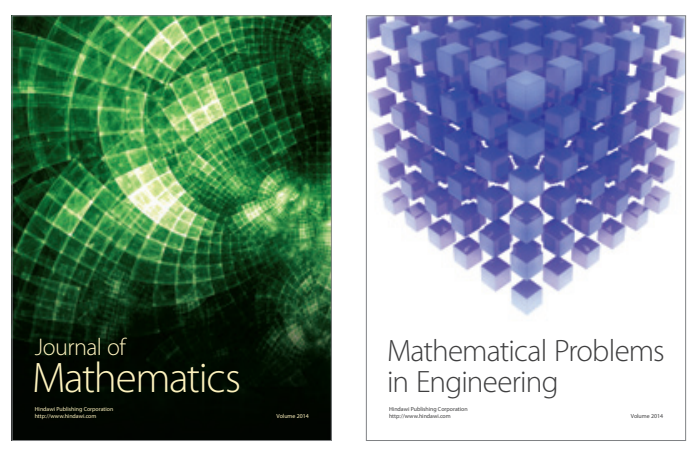

Mathematical Problems in Engineering
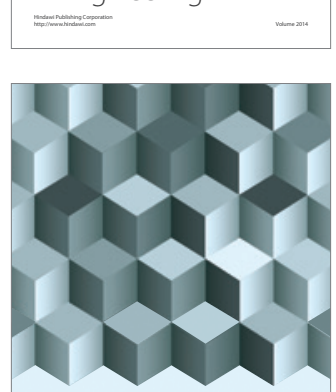

Journal of

Function Spaces
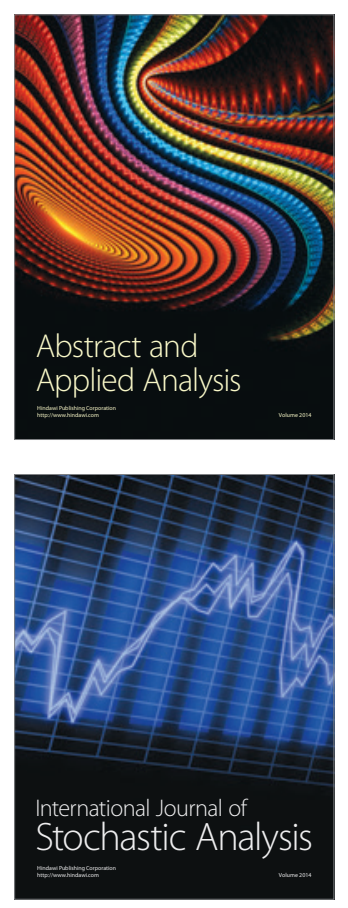

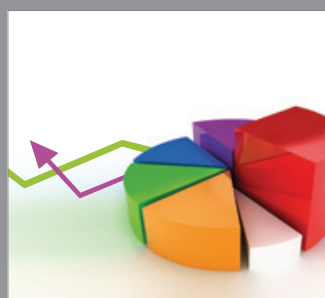

ournal of

Probability and Statistics

Promensencen
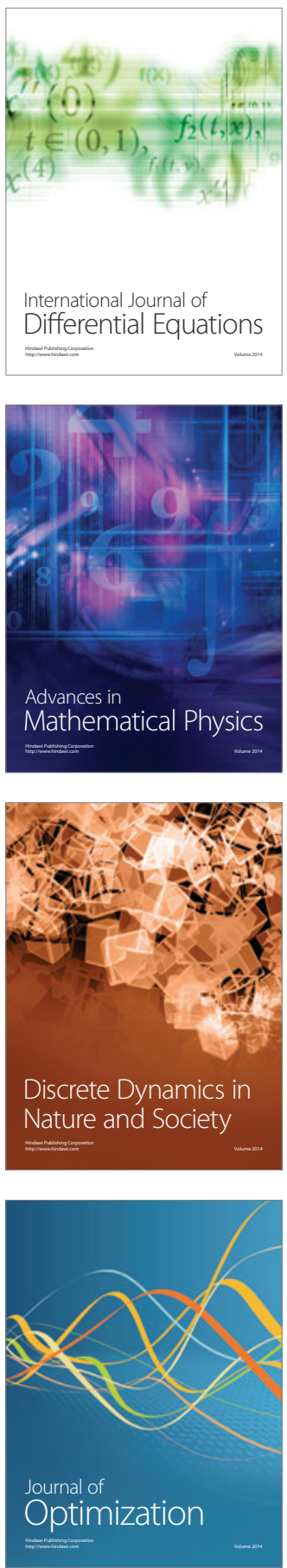